Historic, Archive Document

Do not assume content reflects current scientific knowledge, policies, or practices. 



\section{SPRING 1928 SURPLUS LIST}

ONE-YEAR SEEDLINGS

5,000 Ampelopsis veitchi (Boston Ivy), No.

10,000 Ampelopsis veitchi Bost on lvy),

10,000 Aristolochia sipho (Dutehmans-pipe), No.

12,000 Aristolochia sipho (Dutchmans-pipe), No
3,000 Cytisus laburnum (Laburnum vulgare).

9,000 Berberis thunbergi (Japanese Barberry), $3-6$ in

10,000 Berberis thunbergi (Japanese Barberry), 6-9 in.

14,000 Berberis thunbergi (Japanese Barberry), nursery run.

2,000 Berberis thunbergi (Japancse Barberry), 2 yr., 9-12 in

0,000 Berberis thunbergi (Japanese Barberry) 2 yr., N. R.

$100 \quad 1000$

$\$ 3.00 \$ 20.00$

$\begin{array}{ll}1.00 & 10.00 \\ 2.00 & 15.00\end{array}$

$\begin{array}{ll}3.00 & 25.00 \\ 1.50 & 10.00\end{array}$

$2.00 \quad 15.00$

$\begin{array}{ll}1.50 & 10.00 \\ 1.00 & 7.50\end{array}$

2.50020 .00

EVERGREENS-One-Year Seedlings

20,000 Picea excelsa (Norway Spruce) ...........................

20,000 Picea pungens glauca (Color

70,000 Thuja occidentalis (American Arborvitae)

9,000 Thuja orientalis (Chinese Arborvita

ct Chinese Arborvitae)

14,000 Thuja orientalis aurea (Golden Chinese Arborvitae)

4,000 Abies balsamea (Balsanl in)

, 000 Abies firma

9,000 Abies fraseri (Fraser Fir) ......................

14,000 Chamaecyparis obtusa (Hinoki Cypres)

10,000 Pinus banksiana (Jack Pine).

25,000 Pinus densiflora (Japanese

4,000 Pinus jeffreyi (Jeffrey Pine).

18,000 Pinus nigra (Austrian Pine)

18,000 Pinus nigra (Austrian Pine)

20,000 Pinus sylvestris (Scotch Pine)

12,000 Picea alba (White Spruee)

15,000 Picea canadensis (Blaek Hills Siruee)

7,000 Pseudotsuga douglasi (Douglas-fir)

$5,000)$ Tsuga mertensiana (Mountain Hemlock
25,000 Tsuga canadensis (Canada Hemlock)

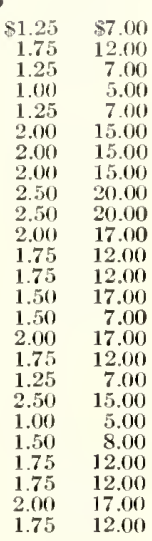

EVERGREENS-Two-Year Seedlings

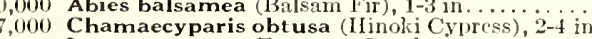

2,000 Larix europaea (European Larch), 4-6 in,

15,000 Picea pungens glauca (Colorado Blue Spruce) i- 3 in

6,000 Picea sitchensis (Sitka Spruce), $3-6$ in
9,000 Picea sitchensis (Sitlia Spruee) $6-8$ in

10,000 Pinus densiflora (Jayanese Red Pine), 3-6 in . . .

20,000 Pinus mughus (Mugho Pine), 1-3 in

12,000 Pinus nigra (Austrian Pine), 3-6 in .

5,000 Pinus resinosa (Red Pine), $2-5$ in.

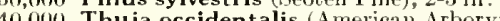

4,000 Thuja occidentalis (Amoen $2-5$ in .

50,000 Thuja occidentalis (American A rborvitae), $2-6$ in....

EVERGREENS-Two-Year, One Transplanted

3,000 Abies brachyphylla (Nikko Fir), 2-5 in.................. $\$ 6.00 \$$

4,000 Picea pungens glauca (Colorado Blue Spruce), 1-3 in............ $4.00 \quad 30.00$

1,000 Picea pungens glauca (Colorado Blue spruce), $3-6$ in.............. $6.00 \quad 50.00$

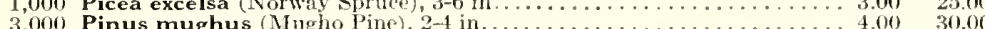

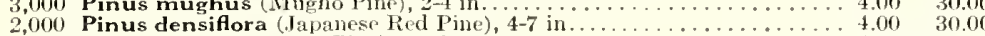

.

500 Thuja occidentalis (American Arborvitae)

700 Thuja orientalis (Chimese Alborvitar).

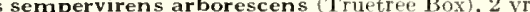

6,000 Buxus sempervirens arborescens (Truetree $\mathrm{Box}$ ), 1 yr.

8,000 Spiraea Gladstone

\section{LINING OUT STOCK}

13,000 Deutzia Pride of Rochester

, 000 Deutzia crenata

2000 Red Snowberry...

700 Philadelphus cor

3,000 Weigela rose

1,000 Weigela desboise

900 Weigela amabili

300 Weigela amabilis....

\section{SHRUBS - Two and Three Years}

300 Deutzia crenata, $4-5 \mathrm{ft}$.
200 Deutzia crenata, $3-4 \mathrm{ft}$.

300) Deutzia crenata, $2-3 \mathrm{ft} \ldots \ldots+1,4+5 \mathrm{ft}$

100 Deutzia Pride of Rochester, $3-4 \mathrm{ft}$

200 Deutzia Pride of Rochester, 2-3 f

200 Forsythia fortunei, $3-4 \mathrm{ft}$

60 .

200 Spiraea opulifolia aurea, $4-5 \mathrm{ft}$

300 Spiraea opulifolia aurea, 3-4

100 Symphoricarpos racemosus (White Snowberry), 2-3 ft

300 Symphoricarpos racemosus (White Snowberry) $18-2 t$ in

100 Weigela amabilis, 4-5 f

150 Weigela amabilis, $3-4 \mathrm{ft}$

100 Weigela amabilis, $2-3 \mathrm{ft}$

250 Weigs a desboisi,

300 Weigela desboisi, 2

40 Weigela floribunda, $2-3 \mathrm{ft}$

100 Philadalphus coronarius, $3-4$

50 Philadelphus coronarius, $2-3 \mathrm{ft}$

Philadelphus grandifiora, $3-4 \mathrm{ft}$

90 Philadelphus grandifiora, $2-3 \mathrm{f}$

All stock well grown and freshly dug

Free Packing for Cash With Order

\section{BROUWER'S NURSERIES}

35 Keeney Lane, NEW LONDON, CONN. 


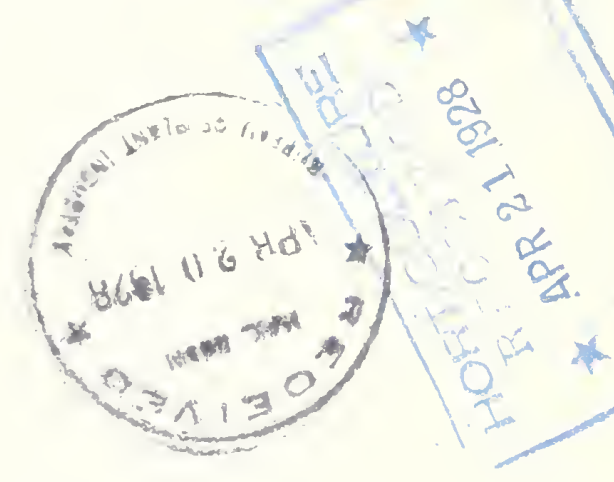

